\title{
Artesunate attenuates proliferation of epithelial cells by downregulating the NF-KB and AKT signaling pathways in benign mammary gland hyperplasia rats
}

\author{
Wei Li, Lina Zhao, Yiliang Li, Zhen Zhai \\ Department of Breast, Dongfang Hospital Beijing University of Chinese Medicine, Beijing, China \\ Contributions: (I) Conception and design: All authors; (II) Administrative support: None; (III) Provision of study materials or patients: None; (IV) \\ Collection and assembly of data: W Li, Y Li, Z Zhai; (V) Data analysis and interpretation: All authors; (VI) Manuscript writing: All authors; (VII) \\ Final approval of manuscript: All authors. \\ Correspondence to: Lina Zhao. Department of Breast, Dongfang Hospital Beijing University of Chinese Medicine, 6 Fangxingyuan, Fengtai District, \\ Beijing 100078, China. Email: linalucky2010@126.com.
} Background: The aim of this study was to investigate the effects of artesunate (ART) on breast epithelial
cell proliferation in vitro and in vivo.

Methods: Immortalized human non-cancer mammary epithelial (MCF-10A) cells were used to determine the effect of ART on estrogen-induced mammary hyperplasia cells. We investigated the effect of ART on the synthesis of cyclooxygenase-2 (COX-2) and proliferating cell nuclear antigen (PCNA) in MCF-10A by treating MCF-10A $36 \mathrm{~h}$ with different concentrations of ART (0, 100, 200, $400 \mu \mathrm{m}, \mathrm{n}=12$ /group). We then investigated the effect of ART on estrogen induced COX-2, PCNA, nuclear factor-kappa B (NF- $\kappa \mathrm{B})$, and pNF- $\kappa B$ synthesis by treating MCF-10A with both estrogen and ART (0, 50, 100, $200 \mu \mathrm{m}, \mathrm{n}=12$ /group). A mammary hyperplasia model $(\mathrm{MGH})$ was established in rats. All rats $(\mathrm{n}=12)$ were divided into 4 groups [group A: negative control $(\mathrm{NC})+$ Art -; group B: NC + Art +; group C: $\mathrm{MGH}+$ Art -; group D: MGH + Art +] by the random number table method and the effects of ART on estradiol-induced mammary hyperplasia, fibrosis, and phosphorylation of $\mathrm{AKT}$ and $\mathrm{NF}-\kappa \mathrm{B}$ were studied by histopathological staining, Masson trichrome staining, immunohistochemistry (IHC), and western blotting.

Results: The proliferation and inflammation of mammary epithelial cells were blocked by ART $(\mathrm{P}<0.05)$. The phosphorylation of NF- $\kappa \mathrm{B}$ induced by estradiol in MCF-10A was attenuated by ART $(\mathrm{P}<0.05)$. In the rat $\mathrm{MGH}$, ART reduced cell proliferation and fibrosis $(\mathrm{P}<0.05)$ and inhibited the phosphorylation of AKT and $\mathrm{NF}-\kappa \mathrm{B}(\mathrm{P}<0.05)$.

Conclusions: The drug ART inhibits estrogen-induced breast hyperplasia by blocking AKT and NFkB phosphorylation.

Keywords: Artesunate (ART); traditional Chinese medicine; benign mammary gland hyperplasia; nuclear factorkappa B (NF- $\mathrm{B})$

Submitted Mar 07, 2021. Accepted for publication Apr 28, 2021.

doi: $10.21037 / \mathrm{atm}-21-1447$

View this article at: http://dx.doi.org/10.21037/atm-21-1447

\section{Introduction}

Hyperplasia is a benign mammary condition that is usually clinically occult. Clinical manifestations of mammary gland hyperplasia are nonspecific, including pain and local lump. Generally, hyperplasia is most likely to occur in women aged $>35$ years, and it can be found in women of all age groups (1). Although benign hyperplasia is not a malignancy, it shares a variety of activated signaling with breast cancer. Additionally, similar to most cancers, benign mammary epithelium grows continuously; there have been no effective 
agents discovered that can control cell proliferation, and surgical treatment is usually indicated. The imbalance of hormone levels and the abnormal expression of hormone receptors in breast tissues are the main causes of breast hyperplasia, causing abnormal cell signaling activation and inducing cell proliferation (1).

Artesunate (ART) is an important artemisinin derivative, and its metabolite dihydroartemisinin is most commonly used in the treatment of malaria (2). In recent years, relevant studies have found that ART has a certain effect on tumor treatment (3). Its mechanism mainly involves tumor cell apoptosis, anti-immunosuppression, iron-mediated free radical formation, cell cycle blocking, anti-angiogenesis (4), reversing drug resistance, and restoring chemosensitivity $(5,6)$. At present, a large number of studies on the antitumor activity of ART are underway. Li et al. found that ART can target nasopharyngeal carcinoma by inhibiting the AKT/mTOR pathway (7). Xiao et al. also found that ART can treat oral tongue squamous cell carcinoma by inhibiting AKT (8). Besides, ART has been demonstrated to regulate nuclear factor- $\mathrm{KB} \mathrm{NF}-\kappa \mathrm{B}$ (9), and it has also been shown to have potential in the treatment of breast cancer (10). However, ART has not yet been studied for the treatment of breast hyperplasia.

Although mammary gland hyperplasia is not an inflammatory disease, the continuously growing mass usually causes swelling pain, and tingling at the original site. Some patients may even suffer from itch and fever in the breast. Cyclooxygenase-2 (COX-2) is suggested to generate prostaglandins, which may cause fever, pain, and inflammation (11). Studies by Visscher et al. have confirmed that COX-2 is associated with the proliferation of breast cells (12) and the risk of breast cancer (13). Additionally, NF- $\kappa \mathrm{B}$ affects proinflammatory signaling pathways by regulating COX-2 genes (14). Brantley et al. confirmed that NF- $\kappa B$ plays an important role in regulating the proliferation, branching, and maintenance of the normal epithelial structure of mammary epithelium (15). However,

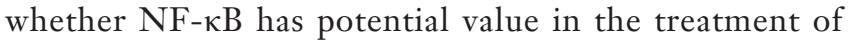
breast hyperplasia still requires further study.

Protein kinase B or Rac, now known as AKT, is implicated in NF- $\mathrm{KB}$ and regulating biological processes in tumors $(16,17)$, and is a kind of serine/threonine-protein kinase $(18,19)$. It is recruited to the plasma membrane as the inactive cytosolic protein. Also, AKT can be activated via serine 473 and threonine 308 phosphorylation responding to cytokines or growth factors through the phosphatidylinositol 3,4,5-triphosphate $\left(\mathrm{PIP}_{3}\right)$ and phosphatidylinositol-3-kinase (PI3K) pathway (20). The phosphorylated AKT blocks some pro-apoptotic proteins, such as Bcl-2 associated agonist of cell death (Bad) and Bcl-2 associated X protein (Bax) (21). Moreover, suppression of AKTinduced apoptosis may inhibit mammary hyperplasia (22).

This study aimed to investigate the effects of ART on breast epithelial cell proliferation in vitro and in vivo.

We present the following article in accordance with the ARRIVE reporting checklist (available at http://dx.doi. org/10.21037/atm-21-1447).

\section{Methods}

\section{Cell culture and treatment}

Immortalized human non-cancer mammary epithelial (MCF-10A) cells, purchased from the American Type Culture Collection (ATCC) (LGC standards, Middlesex, UK), were cultivated with Dulbecco's Modified Eagle Medium (DMEM) 1640 (phenol red-free) supplemented with $5 \%$ fetal bovine serum (FBS), $0.5 \%$ streptomycin, and $0.5 \%$ penicillin in a humidified incubator containing $5 \%$ $\mathrm{CO}_{2}$ at $37^{\circ} \mathrm{C}$. To determine the effects of ART on estradiolinduced breast proliferation, cells were starved using the FBS-free DMEM 1640 for 24 h, and treated with estradiol for various times, either with or without ART.

\section{Chemicals and antibodies}

Antibodies against proliferating cell nuclear antigen (PCNA), COX-2, pNF-кB, NF-кB, AKT, pAKT, and glyceraldehyde 3-phosphate dehydrogenase (GAPDH) were provided by Santa Cruz Biotechnology (Dallas, TX, USA). The ART was provided by the Kunming medicine group (Kunming, YN, China). Estradiol was purchased from Tocris Bioscience (Minneapolis, MN, USA). The remaining antibodies utilized in the current work were provided by Cell Signaling Technology (Danvers, MA, USA).

\section{Animals}

We established a rat model of mammary gland hyperplasia. A total of 12 rats were divided into 4 groups with 3 rats in each group. In group $\mathrm{A}$, the rats were given normal saline injection $(0.1 \mathrm{mg} / \mathrm{kg})$ once a day for 30 days. On group $\mathrm{B}$, the rats were given normal saline injection $(0.1 \mathrm{mg} / \mathrm{kg})$ and ART once a day for 30 days. In group C, estradiol benzoate $(0.5 \mathrm{mg} / \mathrm{kg})$ was injected intramuscularly once 
a day for 25 days, and then progesterone $(4 \mathrm{mg} / \mathrm{kg}$ ) was injected intramuscularly once a day for 5 days. In group D, estradiol benzoate $(0.5 \mathrm{mg} / \mathrm{kg})$ was injected intramuscularly once a day for 25 days, and then progesterone $(4 \mathrm{mg} / \mathrm{kg}$ ) was injected intramuscularly once a day for 5 days. The ART was administered intramuscularly for 30 days, once a day, at the same time. The mammary gland tissues were harvested 1 month after intervention for subsequent analyses. The rats were kept within an approved animal facility. The researchers were not aware of group allocation. Experiments were performed under a project license (No.: JDF-IRB-2014036302) granted by Institutional Animal Care and Use Committee of Dongfang Hospital Beijing University of Chinese Medicine, in compliance with institutional guidelines for the care and use of animals.

\section{Western blotting}

A radioimmunoprecipitation assay (RIPA) buffer was used to lyse the cells and tissue, and western blotting assay was conducted according to the previous description (4). Cells were first incubated with primary antibodies against GAPDH (1:10,000, Sigma, St. Louis, MO, USA) or COX2 (1:500, R\&D Systems, Minneapolis, MN, USA), and then incubated with the horseradish protein (HRP)-conjugated anti-mouse or anti-rabbit secondary antibody $(1: 5,000$, ICN Amersham Pharmacia, Piscataway, NJ, USA). An enhanced chemiluminescence system (Amersham Pharmacia) was used for visualization.

\section{Masson staining and immunobistochemistry (IHC)}

The harvested mammary gland tissues were fixed with neutral buffered formalin and sliced into $5 \mu \mathrm{m}$ sections. Histopathological staining, Masson trichrome staining, and IHC analysis were performed. Then, the sections were blocked using rabbit serum (amphiregulin) or goat serum (COX-2 and EP2). The anti-collagen I, anti-collagen III, and anti-COX-2 (Cayman Chemical, R\&D Systems) were added for incubation. Later, 3,3V-diaminobenzidine (DAB) and Vectastain avidin-biotin complex reagent were added for color development.

\section{Densitometry analysis}

Semiquantitative analyses on various proteins were performed by ImageJ (National Institutes of Health, Bethesda, MD, USA). The band intensity (density) was determined based on the band pixel value and area. The quantitative results were presented in the manner of the ratio of the target protein to the loading control (the housekeeping protein).

\section{Statistical methods}

The software SPSS 22.0 (IBM Corp., Armonk, NY, USA) was used to perform statistical analysis. The values were expressed as means \pm standard deviation $(\mathrm{m} \pm \mathrm{SD})$, and analyzed by one-way analysis of variance (ANOVA). Tukey's test was used to compare multiple means, and Student's $t$-test was utilized to determine the heterogeneities of 2 groups. Values of $\mathrm{P}<0.05$ indicated statistical significance.

\section{Results}

\section{ART blocks the proliferation and inflammation of mammary epithelial cells}

Given that proliferation and inflammation are often associated with mammary gland hyperplasia, we tested the expression levels of COX-2 and PCNA within the MCF$10 \mathrm{~A}$ cells. The epithelial cells were exposed to phosphate buffered saline (PBS), 100, 200, and $400 \mu \mathrm{M}$ ART in a $10 \%$ serum cultured medium. As indicated in Figure 1A, treatment with ART decreased the expression of COX2 and PCNA in a dose-dependent manner, and the most remarkable decreasing effect was observed at $400 \mu \mathrm{M}$ (Figure $1 B, C, \mathrm{COX}-2: 400$ vs. $0 \mu \mathrm{M}=0.82$ vs. 3.42, $\mathrm{P}<0.001$; PCNA: 400 vs. $0 \mu M=1.99$ vs. $2.85, \mathrm{P}<0.05)$.

\section{ART attenuates phosphorylation of NF- $\mathrm{\kappa B}$ induced by estradiol in MCF-10A}

Estradiol can induce breast hyperplasia, and the AKT and NFкB pathways play an important role. In addition, AKT and NFкB can also be regulated by ART. To further confirm the effect and mechanism of ART on estradiol-induced mammary hyperplasia, we treated MCF-10A cells with estradiol and ART. As shown in Figure 2A, low expression levels of COX-2 and PCNA were detected in the Control group, while estradiol treatment significantly elevated the expression levels of these proteins. Administration of ART suppressed the protein expressions in a dose-dependent manner, and $200 \mu \mathrm{M}$ achieved the most obvious inhibition (Figure 2B,C, COX-2: 200 vs. $0 \mu \mathrm{M}$ vs. Control $=1.56$ vs. 6.32 vs. 1.04, $\mathrm{P}<0.001$; PCNA: 200 vs. $0 \mu \mathrm{M}$ vs. Control $=2.18$ 
A

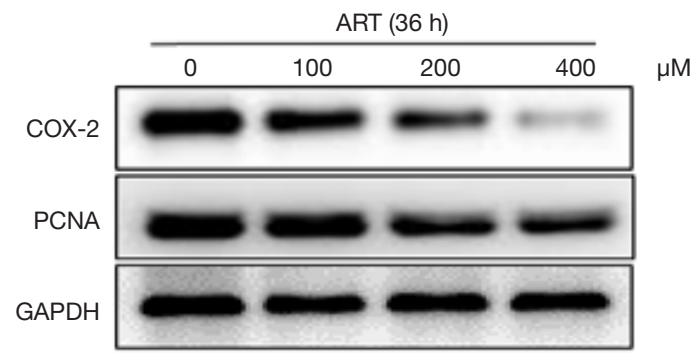

B

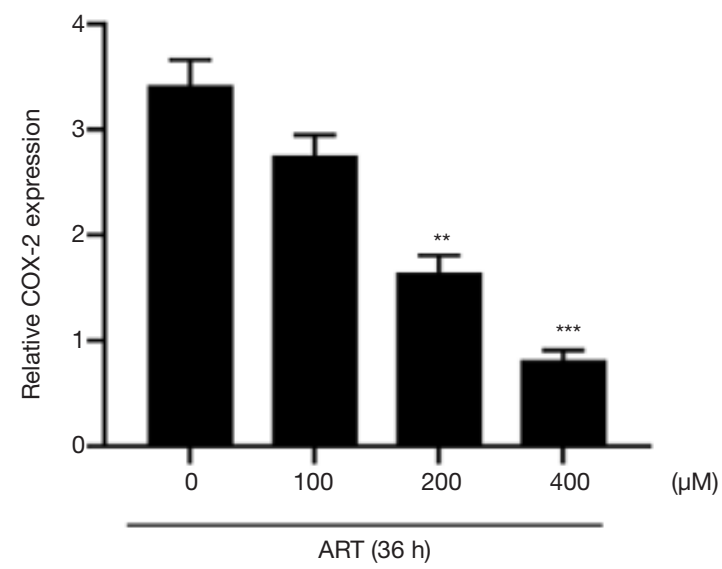

C

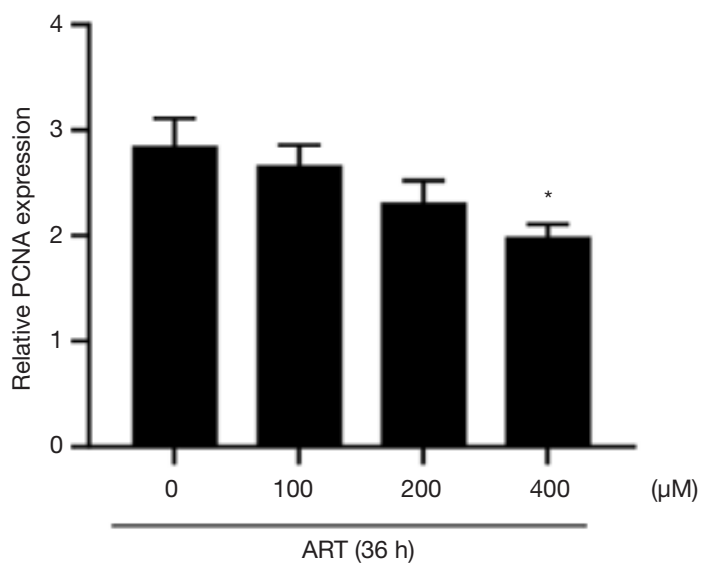

Figure 1 ART suppresses cell proliferation and inflammation in mammary epithelial cells. (A) Typical western blot bands of COX-2 and PCNA at different ART concentrations (0, 100, 200, $400 \mu \mathrm{M})$ for $36 \mathrm{~h}$. (B) Statistical results of the COX-2 expression at different ART concentrations $(0,100,200,400 \mu \mathrm{M})$ for $36 \mathrm{~h}$. (C) Statistical results of the PCNA expression at different ART concentrations $(0,100,200$, $400 \mu \mathrm{M})$ for $36 \mathrm{~h}$. Each protein was quantified according to densitometry and normalized based on GAPDH. The data were expressed as means $\pm \mathrm{SD}(\mathrm{n}=6) .{ }^{*} \mathrm{P}<0.05,{ }^{* *} \mathrm{P}<0.01$, and ${ }^{* * *} \mathrm{P}<0.001$ vs. ART $=0$. ART, artesunate; COX-2, cyclooxygenase-2; PCNA, proliferating cell nuclear antigen; GAPDH, glyceraldehyde 3-phosphate dehydrogenase; SD, standard deviation.

vs. 4.72 vs. 1.01, $\mathrm{P}<0.001)$. Estradiol treatment elevated the phosphorylation of NF- $\kappa \mathrm{B}$, which was suppressed by $200 \mu \mathrm{M}$ ART (Figure 2D). Moreover, ART alleviated the activation of NF- $\kappa B$ pathways in mammary epithelial cells (Figure $2 E, \mathrm{pNF}-\kappa \mathrm{B}: 200$ vs. $0 \mu \mathrm{M} v s$. Control =1.48 vs. 5.74 vs. 1.01, $\mathrm{P}<0.001)$.

\section{ART reduces cell proliferation and fibrosis in estradiol- induced mammary gland byperplasia}

To determine the effects of ART on mammary gland hyperplasia in vivo, we constructed an animal model of mammary gland hyperplasia using rats.We first confirmed that the expression of COX-2 was significantly increased in the rat mammary hyperplasia model (MGH), while ART treatment reduced COX-2 expression by $50 \%$ (Figure $3 A, B$,
COX-2: $\mathrm{NC}+$ normal saline vs. $\mathrm{NC}+$ ART $v s . \mathrm{MGH}+$ normal saline vs. MGH + ART =1.07 vs. 0.62 vs. 6.92 vs. $3.46, \mathrm{P}<0.001)$. Then, we examined the expression levels of Bax in the mammary gland tissues. Bax was significantly down-regulated in breast hyperplasia. It was shown that ART could reverse the expression of Bax (Figure 3A,C).

Blue fibrils were mostly present within the interstitial breast tubules, according to results of Masson trichromepositive staining. Typically, ductal hyperplasia accompanying circular changes serves as the distinct sign to distinguish from the regular and diffuse enlargement and rounding of nuclei under hormone therapy (black arrow indicated). The fibrils remarkably increased within the breast tissue in mammary gland hyperplasia rats and decreased after ART administration (Figure 3D). Semiquantitative analyses of IHC revealed that the COX-2 positive area in the breast 
A

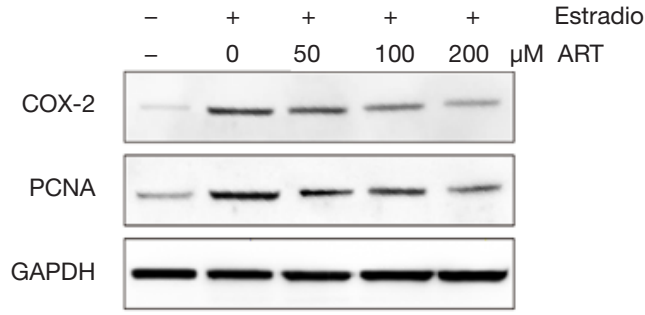

B

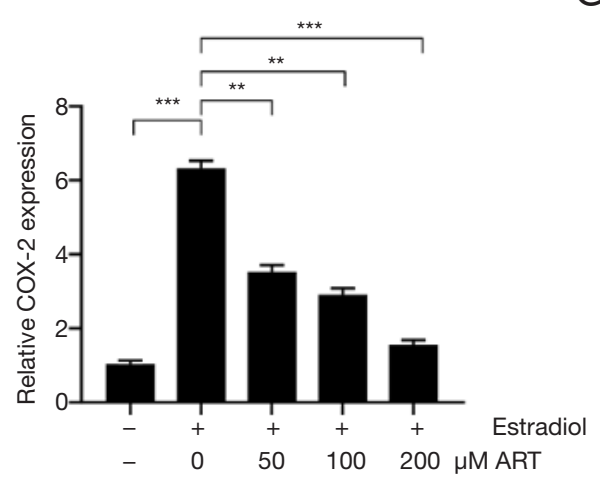

$\begin{array}{lcccccc}\mathrm{D} & - & + & + & + & + & \text { Estradiol } \\ & - & 0 & 50 & 100 & 200 & \mu \mathrm{M} \text { ART }\end{array}$

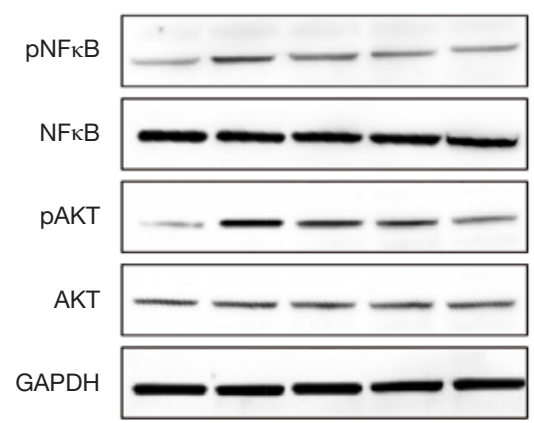

C

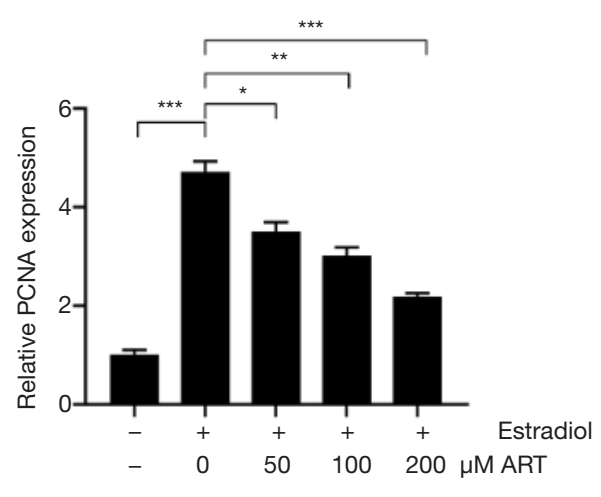

$\mathrm{E}$

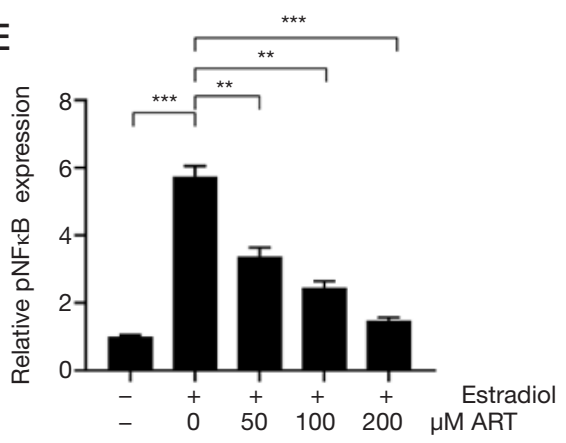

Figure 2 ART suppresses the phosphorylations of NF- $\mathrm{kB}$ in estradiol-induced MCF-10A. (A) Typical western blot bands of COX-2 and PCNA at estradiol and different ART concentrations $(0,50,100,200 \mu \mathrm{M})$ for $24 \mathrm{~h}$. (B) Statistical results of the COX-2 expression at estradiol and different ART concentrations $(0,50,100,200 \mu \mathrm{M})$ for $24 \mathrm{~h}$. (C) Statistical results of the PCNA expression at estradiol and

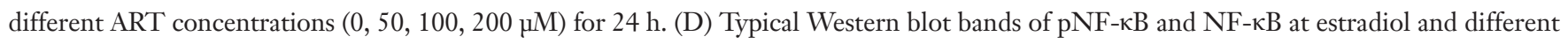

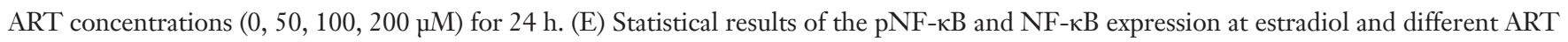
concentrations $(0,50,100,200 \mu \mathrm{M})$ for $24 \mathrm{~h}$. Each protein was quantified according to densitometry and normalized based on GAPDH. The data were expressed as means $\pm \mathrm{SD}(\mathrm{n}=6) .{ }^{*} \mathrm{P}<0.05,{ }^{* *} \mathrm{P}<0.01$, and ${ }^{* * *} \mathrm{P}<0.001$ vs. ART- and estradiol-. NF- $\mathrm{kB}$, nuclear factor-kappa $\mathrm{B}$; MCF-10A, immortalized human non-cancer mammary epithelial; ART, artesunate; COX-2, cyclooxygenase-2; PCNA, proliferating cell nuclear antigen; GAPDH, glyceraldehyde 3-phosphate dehydrogenase; SD, standard deviation.

tubule increased by $\sim 8$ fold when compared to the control breast tissues; besides, ART administration significantly down-regulated the COX-2 expression to $50 \%$ (Figure $3 E$, $\mathrm{NC} v$ s. MGH vs. MGH + ART $=1.09$ vs. 3.90 vs. 2.23, $\mathrm{P}<0.01)$. To examine the effects of ART on extracellular protein accumulation following estradiol and progesterone administration, the expression levels of collagen I and III were detected by IHC. According to Figure $3 F, G$, the baseline collagen I and III expressions were detected in the control group, and they were substantially up-regulated 


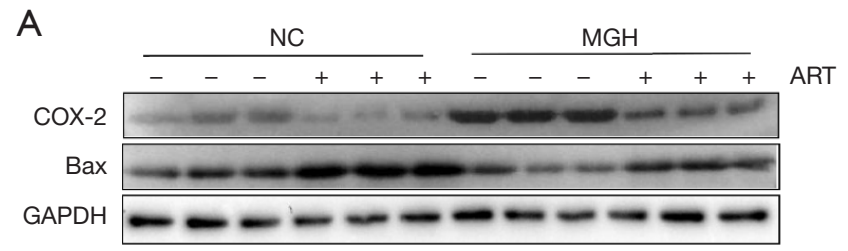

B

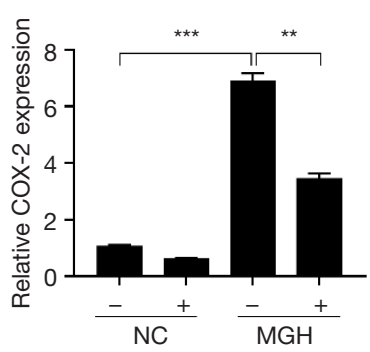

Masson staining (200x)
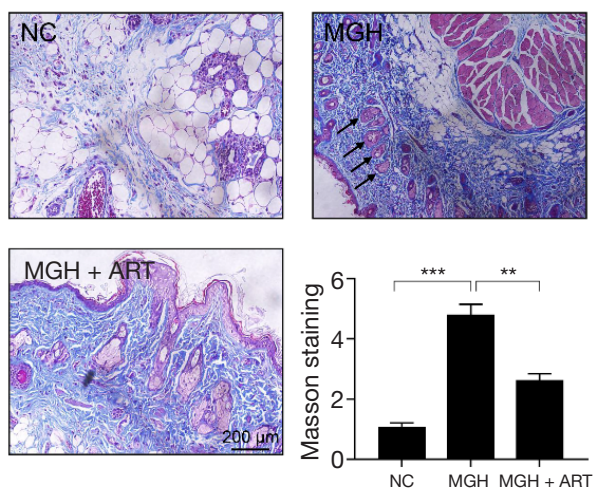

F IHC-Col-1 staining (200x)
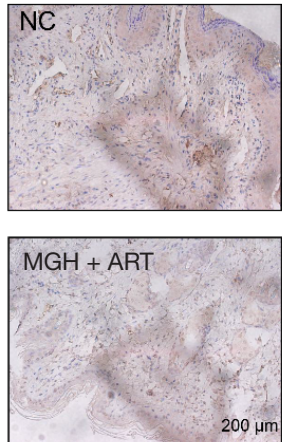

C

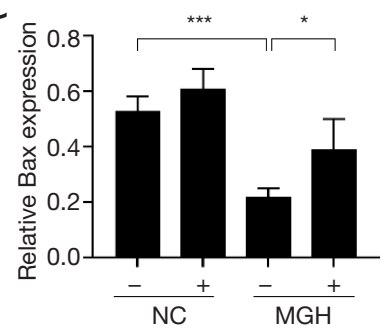

E IHC-COX-2 staining (200x)
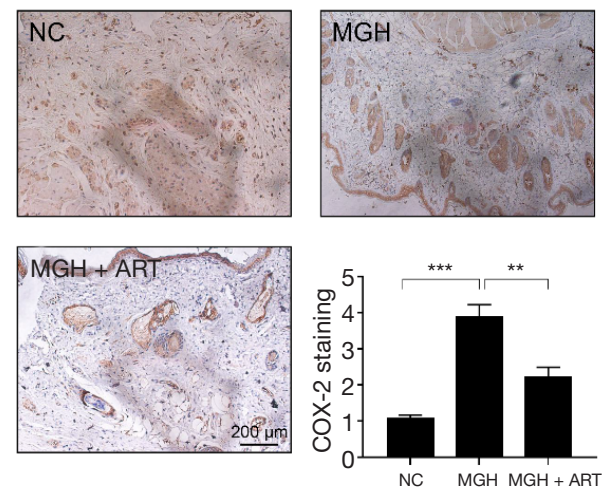

G IHC-Col-3 staining (200x)
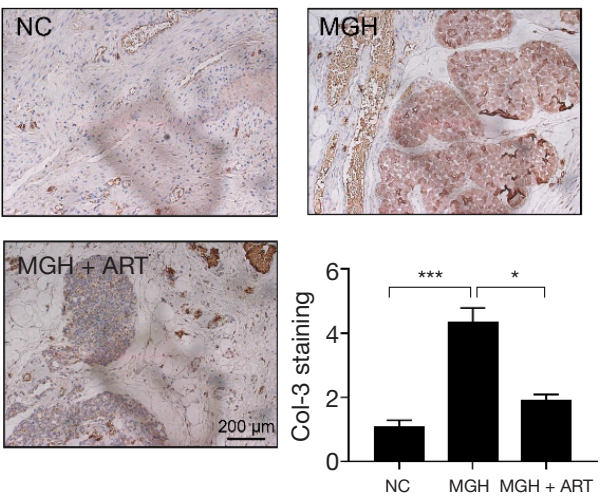

Figure 3 ART attenuates the proliferation and hyperplasia in the hyperplastic breast. (A) Typical western blot bands of COX-2 and Bax of mammary glands in each group. (B) Statistical results of the COX-2 expression of mammary glands in each group. (C) Statistical results of the PCNA expression of mammary glands in each group. (D) Masson staining results of mammary glands in each group. (E) IHCCOX-2 staining results of mammary glands in each group. (F) IHC-Col-1 staining results of mammary glands in each group. (G) IHCCol-2 staining results of mammary glands in each group. Each protein was quantified according to densitometry and normalized based on GAPDH. The data were expressed as means $\pm \mathrm{SD}(\mathrm{n}=6)$. ${ }^{*} \mathrm{P}<0.05$, ${ }^{* *} \mathrm{P}<0.01$, and ${ }^{* * *} \mathrm{P}<0.001$. Bax, Bcl-2 associated $\mathrm{X}$ protein; NF- $\mathrm{kB}$, nuclear factor-kappa B; ART, artesunate; IHC, immunohistochemical; COX-2, cyclooxygenase-2; PCNA, proliferating cell nuclear antigen; GAPDH, glyceraldehyde 3-phosphate dehydrogenase; SD, standard deviation. 
A

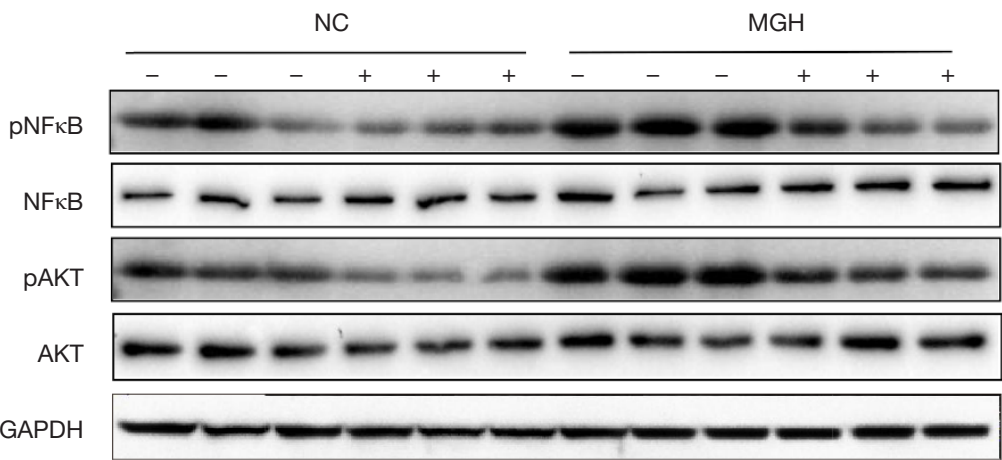

B

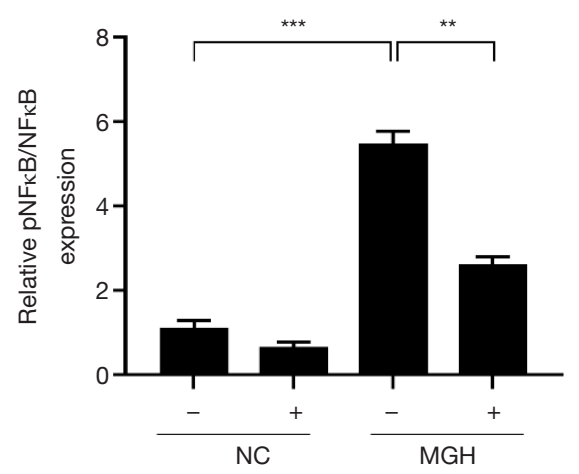

C

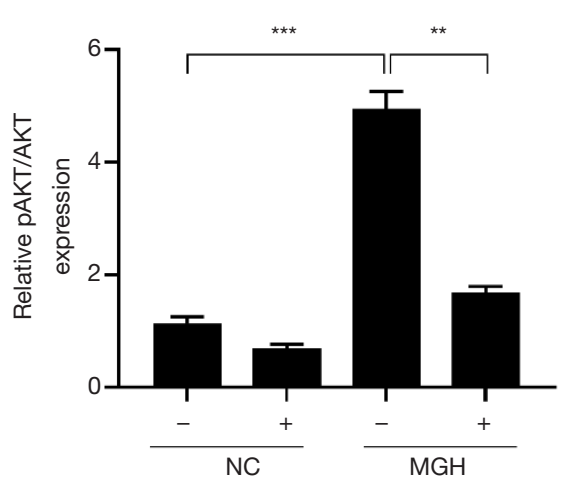

Figure 4 ART suppressed the phosphorylation of AKT and NF- $\mathrm{AB}$ separately in the hyperplastic breast. (A) Typical western blot bands of NF- $\kappa$ B, pNF- $\kappa$ B, AKT, and pAKT of mammary glands in each group. (B) Statistical results of the pNF- $\mathrm{B} / \mathrm{NF}-\kappa \mathrm{B}$ of mammary glands in each group. (C) Statistical results of the pAKT/AKT of mammary glands in each group. Each protein was quantified according to densitometry and normalized based on GAPDH. The data were expressed as means $\pm \mathrm{SD}(\mathrm{n}=6) .{ }^{* *} \mathrm{P}<0.01$, and ${ }^{* * *} \mathrm{P}<0.001$. NF- $\mathrm{B}$, nuclear factor-kappa B; ART, artesunate; GAPDH, glyceraldehyde 3-phosphate dehydrogenase; SD, standard deviation.

after estradiol and progesterone treatment. Treatment with ART significantly reversed the expression levels of collagen I and III (Col-1: NC vs. MGH vs. MGH + ART $=1.07$ vs. 3.38 vs. 2.03, $\mathrm{P}<0.05$; Col-3: NC vs. MGH vs. MGH + ART $=1.10$ vs. 4.36 vs. $1.92, \mathrm{P}<0.05)$.

\section{ART inbibits the phosphorylation of AKT and NF- $\mathrm{\kappa B}$ in mammary gland byperplasia}

The above experiments confirmed that ART could inhibit estradiol-induced breast hyperplasia both in vitro and in vivo. To further explore the mechanism of this phenomenon, we examined the expression of AKT and NF$\mathrm{KB}$ in the mammary glands of rats in each group. As shown in Figure 4A,B,C, phosphorylated AKT and NF- $\mathrm{B}$ proteins were slightly expressed in PBS-treated breast tissue with/ without ART treatment, and their expression levels were up-regulated in mammary gland hyperplasia induced by estradiol and progesterone. However, ART administration inhibited estradiol-induced phosphorylation of mammary $\mathrm{AKT}$ and NF-KB. Additionally, neither hormone nor ART treatment affected the expression levels of total AKT and total NF- $\mathrm{NB}$.

\section{Discussion}

Benign hyperplasia of the breast is a disease of the breast caused by the overproliferation of mammary gland tissues. It is estimated that 8 out of 10 women are susceptible to this disease. Hormone imbalance has been considered an independent risk for mammary gland hyperplasia and promotes the development and progression of breast pain. Excessive formation of the glandular tissue and epithelium in the breast is the typical pathological feature. Although ART is effective for inhibiting the expression of COX-2 as well as the proliferation and migration of breast cancer 
cells, its role in benign mammary gland hyperplasia remains unknown. In this study, we demonstrated that ART blocked $\mathrm{AKT}$ and NF- $\mathrm{KB}$ signaling and inhibited the proliferation in the hyperplastic mammary gland. Administration of ART also attenuated the breast tubule interstitial fibrosis and extracellular deposition in rats with mammary gland hyperplasia. These data suggest that ART is a potential breast hyperplasia inhibitor.

Several Chinese patients with mammary gland hyperplasia are acutely aware of potential side effects induced by synthetic drugs. As a result, they prefer alternative treatments, such as traditional Chinese medicine. The drug ART is a kind of antimalarial agent isolated from Artemisia Annua, the sweet wormwood plant. The derivative, ART, a sesquiterpene lactone (a compound constituted by 3 isoprene units that are bound onto the cyclic organic esters), is obtained by distilling the Artemisia Annua flower clusters or dried leaves (23). This plant possesses feverreducing (antipyretic) effects, which were initially identified in the 4th century CE by Chinese physicians, and this plant was then called Qinghao, and used as the natural remedy called Qinghao tea. On the one hand, the Qinghao remedy is usually used to treat malaria and hemorrhoids. On the other hand, ART has been proved to inhibit kinds of cancers, including breast cancer (24). Prostaglandin $\mathrm{E}_{2}$ (PGE2) serves as a pain indicator, and is also the primary eicosanoid produced via the COX-2 pathway within the mammary gland (25). The COX-2 exerts a vital role during the development of the breast at the early stage (26). Injection of estradiol causes COX-2 overexpression which in turn causes mammary hyperplasia in humans and mice. Although the elevated progression of breast epithelial cells has been considered to be associated with mammary gland hyperplasia, it has been debated whether it is an inflammatory disease. Our results showed a significant increase in COX-2 expression in excessive hormoneinduced female rats' breasts. The underlying mechanism of inflammatory signaling overactivation in promoting mammary gland hyperplasia should be further studied. The human normal breast epithelial cell line was used, which revealed that excessive estradiol treatment dramatically increased the cell proliferation in phenol-free red medium. Meanwhile, inhibiting the activation of NF- $\mathrm{kB}$ within the mammary glands in mice resulted in reduced lobuloalveolar proliferation during pregnancy (27), and meanwhile, elevating the activity of NF- $\kappa B$ resulted in hyperplasia of the mammary gland in vivo (1). Our results suggested that ART suppressed the activity of NF- $\mathrm{KB}$ signaling in vitro (Figure 2).
Additional analyses in vivo indicated that the $\mathrm{pNF}-\kappa \mathrm{B}$ expression was significantly increased within the estrogentreated mammary gland hyperplasia rats as compared to normal controls (Figure 3). In conclusion, findings in this study suggested the mechanism that ART represses $\mathrm{NF}-\kappa \mathrm{B}$ phosphorylation to reduce cell proliferation and inhibit mammary gland hyperplasia. Nonetheless, NF$\kappa \mathrm{B}$ serves as the multifunctional transcription factor and the NF- $\mathrm{KB}$ signaling pathway cross-talks with numerous other pathways in the regulation of mammary gland hyperplasia. More experiments are needed to illustrate the detailed mechanisms of ART in alleviating mammary gland hyperplasia.

It was indicated that the PI3K/AKT pathway exerts a role in the regulation of tumor genesis. The PI3K serves as a critical AKT activation regulator, many kinases and stimuli (such as estrogen) can promote proliferation of the AKT signaling pathway. As a result, the AKT pathway is activated, finally promoting cell survival and growth. Mutational activation and AKT pathway component amplification participate in the pathogenic causes of numerous cancers. Both Bad and Bax belong to the BCL2 protein family. Over-expressed Bax accelerates cell apoptosis. The activation of growth factors in the PI3K/ AKT signal transduction pathway peaks when $\mathrm{Bad}$ is phosphorylated, thus inhibiting apoptosis and accelerating survival of cells (28). The AKT has been identified as a promising therapeutic target, and the phosphorous AKT expression was found to negatively correlate with mammary gland hyperplasia in the current study. Besides, the efficacy of AKT inhibitors as monotherapies or combined with ART in treating the animal models with mammary gland hyperplasia was also measured. The complicated mechanism following AKT suppression indicated that more treatments should be used in combination to prevent resistance and improve the clinical response. Further studies are required to determine more rational combinations to improve AKT inhibitor efficacy through promoting apoptosis or targeting the compensatory mechanism. The identification of responsive biomarkers is necessary for the development of novel treatments.

Proliferative tissue is related to carcinogenesis; therefore, the mammary gland histology was analyzed in 8-week-old rats to examine the abnormal morphological changes. Based on Masson staining of mammary glands in rats, discontinued ductal epithelium and mild focal hyperplasia were observed in comparison with littermate controls. None of the rats had developed tumors at 2 months old. 
Fibroblasts often occur near tumors and contribute to malignancy; however, a previous study has revealed that fibroblasts and myoepithelium proliferation contribute very little to stromal tissues (27). That means that fibrosis may play an important role in the benign pathogenesis of mammary gland hyperplasia. Moreover, estrogen was used as a strong fibrotic inducer in cells. In this study, we found that blue-stained collagen fibrils deposited dramatically in the breast stroma, suggesting that excessive estradiolinduced fibrotic signaling could be blocked by ART treatment (Figure 3). Wen et al. found that ART mitigated the fibrosis and inflammation of tubulointerstitial nephritis in rats receiving 5/6 subtotal nephrectomy through the NF- $\mathrm{KB}$ signaling pathway (29). Thus, ART may suppress breast stromal fibrosis and extracellular accumulation by suppressing the activation of the NF- $\kappa B$ pathway. Our study was not without limitations: the mechanisms by which ART affects breast fibrosis remain to be further investigated, meanwhile it is necessary to enlarge the sample size of experiment in the further study.

\section{Conclusions}

In conclusion, our results firstly demonstrated that ART attenuated mammary hyperplasia by suppressing the AKT and $\mathrm{NF}-\kappa \mathrm{B}$ pathways in vitro and in vivo. Our findings indicated that ART may have therapeutic potential for the treatment of ductal fibrosis.

\section{Acknowledgments}

Funding: This study was supported by the "1166" Talent Project-Young and Middle-aged Experts of Dongfang Hospital Beijing University of Chinese Medicine (040204001001002025).

\section{Footnote}

Reporting Checklist: The authors have completed the ARRIVE reporting checklist. Available at http://dx.doi. org/10.21037/atm-21-1447

Data Sharing Statement: Available at http://dx.doi. org/10.21037/atm-21-1447

Conflicts of Interest: All authors have completed the ICMJE uniform disclosure form (available at http://dx.doi. org/10.21037/atm-21-1447). The authors have no conflicts of interest to declare.

Ethical Statement: The authors are accountable for all aspects of the work in ensuring that questions related to the accuracy or integrity of any part of the work are appropriately investigated and resolved. The rats were kept within the approved animal facility, animal experiments were performed under a project license (No.: JDFIRB-2014036302) granted by Institutional Animal Care and Use Committee of Dongfang Hospital Beijing University of Chinese Medicine, in compliance with institutional guidelines for the care and use of animals.

Open Access Statement: This is an Open Access article distributed in accordance with the Creative Commons Attribution-NonCommercial-NoDerivs 4.0 International License (CC BY-NC-ND 4.0), which permits the noncommercial replication and distribution of the article with the strict proviso that no changes or edits are made and the original work is properly cited (including links to both the formal publication through the relevant DOI and the license). See: https://creativecommons.org/licenses/by-nc-nd/4.0/.

\section{References}

1. Sun L, Guo DH, Liu F, et al. A mouse model of mammary hyperplasia induced by oral hormone administration. Afr J Tradit Complement Altern Med 2017;14:247-52.

2. Sagara I, Beavogui AH, Zongo I, et al. Safety and efficacy of re-treatments with pyronaridine-artesunate in African patients with malaria: a substudy of the WANECAM randomised trial. Lancet Infect Dis 2016;16:189-98.

3. Efferth T, Li PC, Konkimalla VS, et al. From traditional Chinese medicine to rational cancer therapy. Trends Mol Med 2007;13:353-61.

4. Zhang LX, Liu ZN, Ye J, et al. Artesunate exerts an antiimmunosuppressive effect on cervical cancer by inhibiting PGE2 production and Foxp3 expression. Cell Biol Int 2014;38:639-46.

5. Jeong DE, Song HJ, Lim S, et al. Repurposing the antimalarial drug artesunate as a novel therapeutic agent for metastatic renal cell carcinoma due to its attenuation of tumor growth, metastasis, and angiogenesis. Oncotarget 2015;6:33046-64.

6. Mukanganyama S, Naik YS, Widersten M, et al. Proposed reductive metabolism of artemisinin by glutathione transferases in vitro. Free Radic Res 2001;35:427-34.

7. Li Q, Ni W, Deng Z, et al. Targeting nasopharyngeal 
carcinoma by artesunate through inhibiting Akt/mTOR and inducing oxidative stress. Fundam Clin Pharmacol 2017;31:301-10.

8. Xiao Q, Yang L, Hu H, et al. Artesunate targets oral tongue squamous cell carcinoma via mitochondrial dysfunction-dependent oxidative damage and Akt/AMPK/ mTOR inhibition. J Bioenerg Biomembr 2020;52:113-21.

9. Verma S, Kumar VL. Attenuation of gastric mucosal damage by artesunate in rat: Modulation of oxidative stress and NFкB mediated signaling. Chem Biol Interact 2016;257:46-53.

10. Greenshields AL, Fernando W, Hoskin DW. The antimalarial drug artesunate causes cell cycle arrest and apoptosis of triple-negative MDA-MB-468 and HER2enriched SK-BR-3 breast cancer cells. Exp Mol Pathol 2019;107:10-22.

11. Williams CS, Mann M, DuBois RN. The role of cyclooxygenases in inflammation, cancer, and development. Oncogene 1999;18:7908-16.

12. Visscher DW, Pankratz VS, Santisteban M, et al. Association between cyclooxygenase-2 expression in atypical hyperplasia and risk of breast cancer. J Natl Cancer Inst 2008;100:421-7.

13. Feriancová M, Walter I, Singer CF, et al. Expression of COX-2, p16, and Ki67 in the range from normal breast tissue to breast cancer. Neoplasma 2020;200731N798.

14. Li B, Lu Y, Yu L, et al. miR-221/222 promote cancer stemlike cell properties and tumor growth of breast cancer via targeting PTEN and sustained $\mathrm{Akt} / \mathrm{NF}-\kappa \mathrm{B} / \mathrm{COX}-2$ activation. Chemico-Biological Interactions 2017;277:33-42.

15. Brantley DM, Chen C, Cook R, et al. Nuclear Factor- B (NF- B) Regulates Proliferation and Branching in Mouse Mammary Epithelium. Mol Biol Cell 2001;12:1445-55.

16. Ghoneum A, Said N. PI3K-AKT-mTOR and NFкB Pathways in Ovarian Cancer: Implications for Targeted Therapeutics. Cancers (Basel) 2019;11:949.

17. Shang Z, Yu J, Sun L, et al. LncRNA PCAT1 activates $\mathrm{AKT}$ and $\mathrm{NF}-\kappa \mathrm{B}$ signaling in castration-resistant prostate cancer by regulating the PHLPP/FKBP51/IKK $\alpha$ complex. Nucleic Acids Res 2019;47:4211-25.

18. Higuchi M, Masuyama N, Fukui Y, et al. Akt mediates

Cite this article as: $\mathrm{Li}$ W, Zhao L, Li Y, Zhai Z. Artesunate attenuates proliferation of epithelial cells by downregulating the NF- $\kappa \mathrm{B}$ and AKT signaling pathways in benign mammary gland hyperplasia rats. Ann Transl Med 2021;9(10):848. doi: 10.21037/ atm-21-1447
Rac/Cdc42-regulated cell motility in growth factorstimulated cells and in invasive PTEN knockout cells. Current Biology 2001;11:1958-62.

19. Manning BD, Cantley LC. AKT/PKB signaling: navigating downstream. Cell 2007;129:1261-74.

20. Khwaja A, Rodriguezviciana P, Wennstrom S, et al. Matrix adhesion and Ras transformation both activate a phosphoinositide 3-OH kinase and protein kinase B/Akt cellular survival pathway. EMBO J 1997;16:2783-93.

21. Datta SR, Dudek H, Tao X, et al. Akt Phosphorylation of BAD Couples Survival Signals to the Cell-Intrinsic Death Machinery. Cell 1997;91:231-41.

22. Debnath J, Walker SJ, Brugge JS. Akt activation disrupts mammary acinar architecture and enhances proliferation in an mTOR-dependent manner. J Cell Biol 2003;163:315-26.

23. Bridgford JL, Xie SC, Cobbold SA, et al. Artemisinin kills malaria parasites by damaging proteins and inhibiting the proteasome. Nat Commun 2018;9:3801.

24. Singh NP, Lai H. Selective toxicity of dihydroartemisinin and holotransferrin toward human breast cancer cells. Life Sci 2001;70:49-56.

25. Chang SH, Liu CH, Conway R, et al. Role of prostaglandin E2-dependent angiogenic switch in cyclooxygenase 2-induced breast cancer progression. Proc Natl Acad Sci U S A 2004;101:591-6.

26. Ghosh M, Ai Y, Narko K, et al. PPARdelta is protumorigenic in a mouse model of COX-2-induced mammary cancer. Prostaglandins Other Lipid Mediat 2009;88:97-100.

27. Nimmo JS, Plummer JM. Ultrastructural studies of fibroadenomatous hyperplasia of mammary glands of 2 cats. J Comp Pathol 1981;91:41-50.

28. Yang E, Zha J, Jockel J, et al. Bad, a heterodimeric partner for Bcl-XL and Bcl-2, displaces Bax and promotes cell death. Cell 1995;80:285-91.

29. Wen Y, Pan M, Lv L, et al. Artemisinin attenuates tubulointerstitial inflammation and fibrosis via the NF- $\kappa \mathrm{B} /$ NLRP3 pathway in rats with 5/6 subtotal nephrectomy. J Cell Biochem 2019;120:4291-300.

(English Language Editor: J. Jones) 